\title{
Spinocerebellar ataxia type 42
}

INSERM

\section{Source}

INSERM. (1999). Orphanet: an online rare disease and orphan drug data base.

Spinocerebellar ataxia type 42. ORPHA:458803

Spinocerebellar ataxia type 42 is a rare, autosomal dominant cerebellar ataxia

characterized by pure and slowly progressive cerebellar signs combining gait instability,

dysarthria, nystagmus, saccadic eye movements and diplopia. Less frequent clinical signs and symptoms include spasticity, hyperreflexia, decreased distal vibration sense, urinary urgency or incontinence and postural tremor. 